\title{
In vitro propagation of muskmelon (Cucumis melo L.) from nodal segments, shoot tips and cotyledonary nodes
}

\author{
S. Parvin, M. Kausar, M. Enamul Haque, M. Khalekuzzaman, B. Sikdar \\ \& M. Asadul Islam* \\ Professor Joarder DNA \& Chromosome Research Laboratory, Department of Genetic \\ Engineering and Biotechnology, University of Rajshahi, Rajshahi 6205, Bangladesh. \\ *Corresponding author: asadgen@ru.ac.bd
}

\begin{abstract}
A rapid and efficient protocol is outlined for in vitro propagation of muskmelon (Cucumis melo L.) Shoot tips, nodal segments and cotyledonary nodes from in vitro grown seedlings were used as explants. The explants were inoculated on MS medium fortified with different combinations and concentrations of growth regulators viz., BAP, NAA, $\mathrm{GA}_{3}$ and IBA for multiple shoot regeneration. Effective result was found on MS medium supplemented with $2.0 \mathrm{mg} / \mathrm{l} \mathrm{BAP}$, in which $90 \%$ and $70 \%$ cultures induced multiple shoots from nodal segments and shoot tip explants, respectively. Whereas, $70 \%$ cultures of cotyledonary nodes were found to induced shoots on MS medium with $1.5 \mathrm{mg} / \mathrm{l} \mathrm{BAP}+0.1 \mathrm{mg} / \mathrm{l} \mathrm{GA}_{3}$. In vitro regenerated shoots were subcultured on half strength MS medium supplemented with different concentrations of IBA and NAA for successful root induction and the effective result (up to $70 \%$ ) was found in medium with $1 \mathrm{mg} / \mathrm{l}$ IBA. Well rooted in vitro grown plantlets were acclimatized in sandy soil, where as $70 \%$ plantlets survived.
\end{abstract}

Keywords: In vitro propagation, nodal segments, shoots tip, cotyledonary nodes and Cucumis melo.

\section{Introduction}

Muskmelon (Cucumis melo L.) is a well known fruit crop under the family Cucurbitaceae, which are grown well on a wide range of soil types. It is very nutritious for health and contains energy (141 kJ-34 kcal), carbohydrates (8.16g), sugars (7.86g) and dietary fiber (0.9g) (source: www.en.wikipedia. org/wiki/ Cantaloupe). It is beneficial for lowering of blood pressure, regulating heart beat, and possibly preventing strokes, reduce the risk of developing kidney stones and age-related bone loss (source: www.healthclub.com). Though it is conventionally propagated by seeds, germination rate of seeds is very low and they are disease susceptible. Moreover, seeds are not cost effective for commercial growers: the seedlings are very susceptible to fungal and bacterial rots that are mainly due to Alternaria tenuis, Envinia sp, Penicillium sp, Cladosporium cucarerinum, Fusarium sp. and Rhizopussto lonifer. In vitro propagation of plantlets could hence be an alternative method for overcoming these constrains. On the other hand, true to parental type plants can be produced by tissue culture. A few reports have been published in the aspect of tissue culture of muskmelon from different explants. Chan \& Lok (2005) have reported generation of muskmelon from nodal segments and Moreno et al., (1985) from cotyledonary leaves. In Bangladesh there is no report on tissue culture of muskmelon. In the present investigation, attempt has been taken to develop a suitable in vitro direct regeneration protocol from nodal segments, shoot tips and cotyledonary nodes of muskmelon. 


\section{Materials and methods}

The study was carried out at Prof. Joarder DNA and Chromosome Research Lab., Department of Genetic Engineering and Biotechnology, University of Rajshahi. Different explants viz., nodal segments, shoot tips and cotyledonary nodes were collected from in vitro grown seedlings of muskmelon (local cultivar) and seeds were collected from local market. The explants were collected carefully as per explants requirement and subsequently transferred to laminar airflow cabinet. These explants were cultured in MS (Murashige \& Skoog, 1962) medium supplemented with $30 \mathrm{~g} / \mathrm{l}$ sucrose, $7 \mathrm{~g} / \mathrm{l}$ solidifying agar. The $\mathrm{pH}$ of the medium was adjusted, in every case, to 5.6-5.8 and $10 \mathrm{ml}$ of medium was taken in each test tube which was covered by non-absorbent cotton plug. After preparation, medium was autoclaved for 21 minutes at the pressure of $1.5 \mathrm{~kg} / \mathrm{cm}^{2}$ at $121^{\circ} \mathrm{C}$. Plant growth regulators were used in different concentrations and combinations as for direct shoot and root induction. BAP (0.5-3 mg/l), NAA (0.1- $0.5 \mathrm{mg} / \mathrm{l})$ and $\mathrm{GA}_{3}(0.1-$ $0.3 \mathrm{mg} / \mathrm{l})$ were used for multiple shoot regeneration. NAA $(0.1-0.5 \mathrm{mg} / \mathrm{l})$ and IBA (0.5- $3.0 \mathrm{mg} / \mathrm{l})$ were used for root induction. The inoculated cultures were kept in dark condition for 24 hrs and then they were transferred to growth chamber, where the fluorescent light intensity was 2500-3000 lux and the room temperature was $25 \pm 2{ }^{\circ} \mathrm{C}$. Subculture was done 21 days intervals and every experiment was repeated twice. Data were recorded after 15 and 21 days of culture. After complete rooting micro plants were acclimatized in sandy soil and established finally in humus soil with careful maintenance.

\section{Results}

Multiple shoot regeneration of muskmelon from nodal segments, shoot tips and cotyledonary nodes were achieved on MS medium supplemented with different concentrations and combinations of BAP, NAA and $\mathrm{GA}_{3}$. Among the different concentrations of BAP $2 \mathrm{mg} / \mathrm{l}$ in MS medium showed the best effect. Ninety percent (90\%), $70 \%$ and $60 \%$ of cultures responded to induce shoots from nodal segments, shoot tips and cotyledonary nodes, respectively. After 15 days the mean number of shoot per culture from nodal segments, shoot tips and cotyledonary nodes were 5.2, 5.2 and 4.2, respectively and after 21 days they were 6.1, 6.2 and 6.2, respectively. After 15 days the mean length of shoot developed from nodal segments, shoot tips and cotyledonary nodes were $3.4,2.7$ and $2.6 \mathrm{~cm}$, respectively. Whereas after 21 days the values were $4.2,3.2$ and $3.1 \mathrm{~cm}$, respectively (Table 1$)$.

In case of the concentrations and combinations of BAP with $\mathrm{GA}_{3}, 1.5 \mathrm{mg} / \mathrm{l} \mathrm{BAP}$ with $0.1 \mathrm{mg} / \mathrm{l} \mathrm{GA}_{3}$ showed the best result for nodal segment, shoot tips and cotyledonary nodes. The shoots induced from nodal segment, shoot tips, and cotyledonary nodes were $70 \%, 70 \%$ and $75 \%$, respectively. After 15 days the mean number of shoots per culture regenerated from nodal segments, shoot tips and cotyledonary nodes were found to be $4.2,4.2$ and 4.4 and after 21 days the values were 6.2, 6.3 and 6.2, respectively. After 15 days the mean length of shoots per culture from nodal segments, shoot tips and cotyledonary nodes were 3.4, 2.6 and 3.3, respectively. 
See table at the end 
For the concentrations and combinations of BAP with NAA, $3 \mathrm{mg} / \mathrm{l} \mathrm{BAP}$ with 0.1 NAA showed the best result, where $80 \%$ explants produced multiple shoots from all categories of explants but mean number of shoot and mean length of multiple shoot were not satisfactory. In vitro raised microshoots were subcultured on half MS medium supplemented with different combinations and concentrations of IBA and NAA. Between the two concentrations 1mg/l IBA showed the best result, where $75 \%$ shoots rooted. After 15 days the mean number of root per shoot was 7.3, and after 21 days it was 8.1. After 15 days the mean length of root in shoots derived from nodal explants was 5.8 and after 21 days it was 6.1 (Table 2). Microplants after rooting were established in sandy soil, where $70 \%$ plantlets were successively survived in natural condition.

Table 2. Effect of MS medium and different concentrations of IBA and NAA in MS medium for root induction from shoot derived form nodal explants of muskmelon.

\begin{tabular}{|c|c|c|c|c|c|c|c|c|}
\hline \multirow{2}{*}{$\begin{array}{c}\text { Hormonal } \\
\text { supplement } \\
\text { (mg/l) } \\
\text { with half } \\
\text { strength MS }\end{array}$} & \multirow{2}{*}{$\begin{array}{c}\text { No. of } \\
\text { explants } \\
\text { inoculated }\end{array}$} & \multirow{2}{*}{$\begin{array}{c}\% \text { of } \\
\text { explants } \\
\text { induced } \\
\text { root }\end{array}$} & \multicolumn{3}{|c|}{ Mean no. of roots per culture } & \multicolumn{3}{|c|}{ Mean length of root $(\mathrm{cm})$} \\
\hline & & & 15 days & 21 days & $\begin{array}{c}30 \\
\text { Days }\end{array}$ & $\begin{array}{c}15 \\
\text { days }\end{array}$ & $\begin{array}{c}21 \\
\text { days }\end{array}$ & $\begin{array}{c}30 \\
\text { Days }\end{array}$ \\
\hline \multicolumn{9}{|c|}{ IBA } \\
\hline 0.5 & 20 & 65 & $6.2 \pm 0.10$ & $7.3 \pm 0.21$ & $8.1 \pm 0.02$ & $4.2 \pm 0.36$ & $4.8 \pm 0.23$ & $5.2 \pm 0.25$ \\
\hline 1.0 & 20 & 75 & $7.3 \pm 0.24$ & $8.1 \pm 0.10$ & $9.2 \pm 0.32$ & $5.8 \pm 0.26$ & $6.1 \pm 0.08$ & $6.9 \pm 0.06$ \\
\hline 1.5 & 20 & 65 & $6.1 \pm 0.14$ & $7.1 \pm 0.03$ & $7.1 \pm 0.14$ & $4.5 \pm 0.01$ & $4.7 \pm 0.15$ & $5.1 \pm 0.14$ \\
\hline 2.0 & 20 & 60 & $5.4 \pm 0.00$ & $6.2 \pm 0.04$ & $7.3 \pm 0.32$ & $4.3 \pm 0.30$ & $4.6 \pm 0.21$ & $4.9 \pm 0.36$ \\
\hline 3.0 & 20 & 50 & $4.2 \pm 0.50$ & $5.1 \pm 0.42$ & $6.2 \pm 0.32$ & $4.2 \pm 0.08$ & $4.4 \pm 0.01$ & $4.5 \pm 0.25$ \\
\hline \multicolumn{9}{|c|}{ NAA } \\
\hline 0.50 & 20 & 40 & $3.0 \pm 0.02$ & $4.2 \pm 0.84$ & $4.2 \pm 0.36$ & $2.0 \pm 0.62$ & $2.90 \pm 0.25$ & $3.6 \pm 0.32$ \\
\hline 1.00 & 20 & 60 & $5.0 \pm 0.32$ & $5.0 \pm 0.62$ & $6.1 \pm 0.14$ & $3.5 \pm 0.02$ & $4.2 \pm 0.01$ & $4.8 \pm 0.11$ \\
\hline 2.00 & 20 & 50 & $4.1 \pm 0.32$ & $5.2 \pm 0.05$ & $5.2 \pm 0.30$ & $2.5 \pm 0.52$ & $3.0 \pm 0.45$ & $4.1 \pm 0.36$ \\
\hline
\end{tabular}

\section{Discussion}

Tissue culture in propagation and its importance in conservation of genetic resources and clonal improvement have been described by Datta \& Datta (1985) and Kukreja et al. (1989). In this investigation, reproducible protocol has been developed for in vitro regeneration of muskmelon from shoot tips, nodal segments and cotyledonery nodes on MS with various concentrations and combinations of different types of growth regulators. Among different combinations, the effective shoot multiplication from all categories of explants used was found in $2.0 \mathrm{mg} / \mathrm{l}$ BAP (Figs. a,b,c,d,e). However, another better result was observed in BAP with NAA $(3.0 \mathrm{mg} / \mathrm{l}+0.1 \mathrm{mg} / \mathrm{l})$. In case of BAP and NAA, multiple shoot was formed with non significant callus developed in nodal explants (Fig. f).

BAP was also found to be effective for in vitro shoot multiplication in Cucumis melo (Chan \& Lok, 2005; Venkateshwarlu, 2012), Vitis vinifera (Fanizza \& Uicciadi, 1988), Garden baslam (Taha et al., 2009) and in pumpkin (Haque et al., 2010). Cytokinin at lower concentration has been reported for multiple shoot induction in different plant species (Kathal et al., 1988; Singh et al., 1996). 

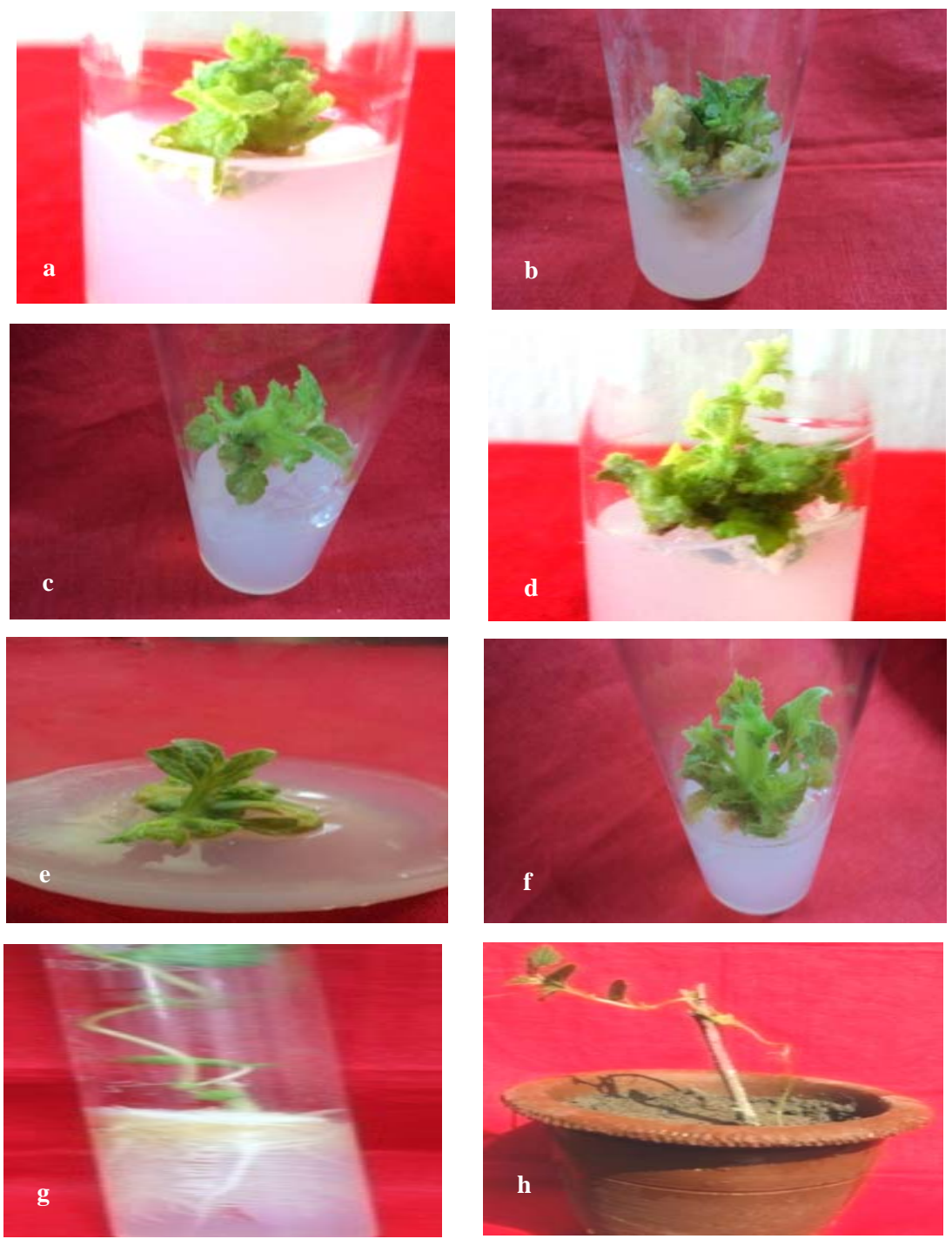

Figure 1. Different stages of in vitro propagation of muskmelon.

a \& b: Shoot multiplication from nodal segment media having 2mg/l BAP.

c \& d: Shoot multiplication from shoot tips media having 2mg/l BAP.

e: Shoot multiplication from cotyledonary nodes media having $2 \mathrm{mg} / \mathrm{l}$ BAP.

f: Shoot multiplication from nodal segment with non significant callus media having BAP with NAA $(3.0 \mathrm{mg} / \mathrm{l}+0.1 \mathrm{mg} / \mathrm{l})$.

g: Root induction from nodal segments media having $1 \mathrm{mg} / \mathrm{l}$ IBA.

h: Hardening of plant. 
Juvenile tissues like cotyledon and various plant parts of seedlings have been reported as good source of explants by a number of workers for a number of plants (Konar \& Oberoi, 1965, Kathal et al., 1988, Singh et al., 1996). Effect of BAP with NAA on multiple shoot induction in different plant species are also in reports (Litz \& Concover, 1981; Novak \& Dolezalova, 1982; Beharav \& Cohen, 1994).

In vitro root induction was conducted on half strength MS with different concentrations of IBA and NAA. Better root induction was observed in IBA (Fig. g). Many researchers reported on root induction in different cucurbitaceous plants using IBA (Kathal et al., 1994; Ahmed and Anis, 2005; Karim et al., 2010; Nahar et al., 2010). Plantlets were successfully established in soil with $70 \%$ survival rate (Fig. h)

A suitable protocol has been established for in vitro direct regeneration. An effective growth regulator has been selected for shoot formation and root regeneration.

\section{Acknowledgements}

The authors are gratefully acknowledged to USDA and MoE of the People's Republic of Bangladesh for their financial support.

\section{References}

Ahmed, N. \& Anis, M. 2005. In vitro Mass propagation of Cucumis sativus L. from nodal segment. Turk Journal Botany, 29: 237-240.

Chan, L.K. \& Lok, K.H. 2005. In vitro plantlets regeneration from nodal segments of muskmelon. Biotechnology, 4: 354-357.

Beharav, A. \& Cohen, Y. 1994. Callus formation from cotyledon and hypocotyls of Cucumis melo L. and Cucumis metuliferus. Cucurbit Genetics Cooperation Report, 17: 88-89.

Datta, P.C. \& Datta, S.C. 1985. Applied biotechnology or medicinal, aromatic and timber plants. Calcutta University: 212-214.

Fanizza, G. \& Uicciadi, L. 1988. The response of a range of genotypes of Vitis vinifera to sequential shoot tip culture at high temperature. Euphytica, 39: 19-23.

Haque, M.E., Rezwana, D., Islam, M.A. \& Sikdar, B. 2010. In Vitro Regeneration of pumpkin (cucurbita maxima) through shoot apical meristem. Journal of Bio-Science, 18: 111-114.

Karim, M.A. \& Ahmed, S.U. 2010. Somatic embryogenesis and micropropagation in teasle gourd. International Journal of Environmental Science and Development, 1(1): 10-14.

Kathal, R., Bhatnagr, S.P. \& Bhojwani, S.S. 1988. Regeneration of plants from leaf explant of Cucumis melo cv. Pusasharbati. Plant Cell Reports, 7: 449-451.

Kathal, S.P., Bhatnagar, S.S. \& Bhojwani, S.S. 1994. Plant regeneration from the callus derived root from explants of Cucumis melo L. cv. Pusasharbati Rekha. Plant Science, 96: 137-142.

Konar, R.N. \& Oberoi, Y.P. 1965. In vitro development of embryoids on the cotyledons of Biota orientalis. Phytomorph, 15: 137- 140. 
Kukreja, A.K., Mathur, A.K., Ahuja, P.S. \&, Thakur R.S. 1989. Tissue culture and biotechnology of medicinal and aromatic plants. Proceedings of an international workshop. CSIR and UNESCO: 83-94.

Litz, R.E. \& Concover, R.A. 1978. Recent advantages in papya tissue culture. Proceeding of the Florida State Horticulture Society, 91: 180-182.

Moreno, V., Sogo, G.M., Granell, I., Garcia, S.B. \& Roig, L.A. 1985. Plant regeneration from calli of melon (Cucumis melo L. cv.“Amarillo Oro”). Plant Cell, Tissue and Organ Culture, 5: 139 - 146.

Murashige, T. \& Skoog, F. 1962. A revised medium for rapid growth and bioassay with tobacco tissue culture. Plant Physiology, 15: 473-497.

Nahar, E., Haque, M.E. \& Sikdar, B. 2010. Comparison of the effects of growth regulators on in vitro regeneration of ridge gourd and sponge gourd through shoot tips. Journal of Bio-science, 18: 93-98.

Novak, J. \& Dolezalova, M. 1982. Hormone control of growth and differentiation in the in vitro cultured tissue of cucumber (Cucumis sativus L.). Biologia (Bratislava), 37: 283-289.

Singh, M.N., Mishra, A.K. \& Bhatnagar, S.P. 1996. In vitro production of plants from cotyledon explant of Cucumis melo L. and their successful transfer to field. Phytomorph, 46: 395-402.

Taha, A., Wagiran, A., Ghazali, H., Huyop, F. \& Parvez, G.K.A. 2009. In vitro regeneration of Garden balsam(Impatiens baslamina) using cotyledons derived seedlings. Biotechnology, 8(1): 44-52.

Venkateshwarlu, M. 2012. Direct multiple shoot proliferation of muskmelon (Cucumis melo L.) from shoot tip explants. International Journal of Pharma and Bio Sciences, 3(2B): 645-652. 
Table 1. Effect of MS medium and different concentrations and combinations of BAP, BAP with GA 3 and BAP with NAA for multiple shoot formation and elongation from nodal explants, shoot tips and cotyledonary nodes of muskmelon.

\begin{tabular}{|c|c|c|c|c|c|c|c|c|c|c|c|c|c|c|c|}
\hline \multirow{3}{*}{$\begin{array}{c}\text { Hormonal } \\
\text { supplements } \\
\text { (mg/l) with } \\
\text { MS }\end{array}$} & \multicolumn{5}{|c|}{ Nodal segments } & \multicolumn{5}{|c|}{ Shoot tips } & \multicolumn{5}{|c|}{ Cotyledonary nodes } \\
\hline & \multirow{2}{*}{\begin{tabular}{|c|}
$\begin{array}{c}\text { \% of } \\
\text { explants } \\
\text { induced } \\
\text { shoots }\end{array}$ \\
\end{tabular}} & \multicolumn{2}{|c|}{$\begin{array}{l}\text { Mean no. of shoot } \\
\text { /culture }\end{array}$} & \multicolumn{2}{|c|}{$\begin{array}{l}\text { Mean length of shoots } \\
\text { /culture }\end{array}$} & \multirow{2}{*}{\begin{tabular}{c|}
$\%$ of \\
explants \\
induced \\
shoots \\
\end{tabular}} & \multicolumn{2}{|c|}{$\begin{array}{c}\text { Mean no. of shoot } \\
\text { /culture }\end{array}$} & \multicolumn{2}{|c|}{\begin{tabular}{|c|}
$\begin{array}{c}\text { Mean length of shoots } \\
\text { /culture }\end{array}$ \\
\end{tabular}} & \multirow{2}{*}{\begin{tabular}{c|}
$\%$ of \\
explants \\
induced \\
shoots
\end{tabular}} & \multicolumn{2}{|c|}{$\begin{array}{c}\text { Mean no. of shoot } \\
\text { /culture }\end{array}$} & \multicolumn{2}{|c|}{$\begin{array}{l}\text { Mean length of shoots } \\
\text { /culture }\end{array}$} \\
\hline & & 15 days & $\begin{array}{c}21 \\
\text { days }\end{array}$ & 15 days & $\begin{array}{c}21 \\
\text { days }\end{array}$ & & 15 days & $\begin{array}{c}21 \\
\text { days }\end{array}$ & 15 days & $\begin{array}{c}21 \\
\text { days }\end{array}$ & & 15 days & $\begin{array}{c}21 \\
\text { days }\end{array}$ & 15 days & $\begin{array}{c}21 \\
\text { days }\end{array}$ \\
\hline \multicolumn{16}{|c|}{ BAP } \\
\hline 0.5 & 15 & $2.2 \pm 0.10$ & $3.1 \pm 0.02$ & $2.1 \pm 0.13$ & $2.9 \pm 0.23$ & 10 & \begin{tabular}{|l|}
$2.2 \pm 0.04$ \\
\end{tabular} & $3.2 \pm 0.30$ & $2.1 \pm 0.32$ & $2.5 \pm 0.11$ & 20 & $2.2 \pm 0.34$ & $3.2 \pm 0.21$ & $2.1 \pm 0.07$ & $2.4 \pm 0.14$ \\
\hline 1.00 & 50 & \begin{tabular}{|l|}
$3.2 \pm 0.02$ \\
\end{tabular} & $4.1 \pm 0.14$ & $2.4 \pm 0.02$ & $3.3 \pm 0.28$ & 30 & $3.3 \pm 0.75$ & $4.2 \pm 0.25$ & $2.2 \pm 0.21$ & $2.7 \pm 0.19$ & 30 & $3.3 \pm 0.22$ & $4.3 \pm 0.14$ & $2.2 \pm 0.49$ & $2.6 \pm 0.28$ \\
\hline 1.5 & 70 & $4.2 \pm 0.23$ & $5.1 \pm 0.22$ & $2.8 \pm 0.15$ & $3.9 \pm 0.41$ & 50 & $4.2 \pm 0.45$ & $5.1 \pm 0.41$ & $2.4 \pm 0.22$ & $2.8 \pm 0.30$ & 40 & $4.2 \pm 0.51$ & $5.4 \pm 0.10$ & $2.4 \pm 0.34$ & $2.6 \pm 0.75$ \\
\hline 2.0 & 90 & $5.2 \pm 0.14$ & $6.1 \pm 0.11$ & $3.4 \pm 0.40$ & $4.2 \pm 0.05$ & 70 & $5.2 \pm 0.02$ & $6.2 \pm 0.21$ & \begin{tabular}{|l|}
$2.7 \pm 0.14$ \\
\end{tabular} & $3.2 \pm 0.12$ & 60 & $4.2 \pm 0.08$ & $6.2 \pm 0.15$ & $2.6 \pm 1.10$ & $3.1 \pm 0.15$ \\
\hline 3 & 60 & $4.3 \pm 0.24$ & $5.2 \pm 0.22$ & $2.9 \pm 0.07$ & $3.5 \pm 0.12$ & 60 & $3.1 \pm 0.19$ & $4.1 \pm 0.11$ & \begin{tabular}{|l|}
$2.5 \pm 0.10$ \\
\end{tabular} & $2.9 \pm 0.09$ & 50 & $2.1 \pm 0.24$ & $3.3 \pm 0.30$ & $2.5 \pm 0.22$ & $2.9 \pm 0.10$ \\
\hline \multicolumn{16}{|c|}{$\mathbf{B A P}+\mathbf{G A}_{3}$} \\
\hline $1+0.1$ & 50 & \begin{tabular}{|l|}
$3.1 \pm 0.21$ \\
\end{tabular} & $5.2 \pm 0.17$ & $2.7 \pm 0.12$ & $3.1 \pm 0.14$ & 55 & \begin{tabular}{|l|}
$3.1 \pm 0.20$ \\
\end{tabular} & $4.2 \pm 0.48$ & $2.4 \pm 0.01$ & $2.7 \pm 0.11$ & 45 & $3.3 \pm 0.31$ & $5.2 \pm 0.15$ & $2.5 \pm 0.09$ & $2.8 \pm 0.11$ \\
\hline $1+0.2$ & 40 & $2.2 \pm 0.09$ & $3.2 \pm 0.12$ & $2.3 \pm 0.22$ & $2.9 \pm 0.11$ & 35 & \begin{tabular}{|l|}
$2.1 \pm 0.47$ \\
\end{tabular} & $3.2 \pm 0.10$ & $2.3 \pm 0.84$ & $2.6 \pm 0.41$ & 30 & $2.2 \pm 0.00$ & $3.3 \pm 0.21$ & $2.3 \pm 0.58$ & $2.6 \pm 0.01$ \\
\hline $1+0.3$ & 30 & $3.3 \pm 0.84$ & $4.1 \pm 0.11$ & $2.1 \pm 0.31$ & $2.7 \pm 0.21$ & 25 & $2.3 \pm 0.41$ & $3.4 \pm 0.23$ & $2.1 \pm 0.04$ & $2.4 \pm 0.11$ & 30 & $3.1 \pm 0.74$ & $4.1 \pm 0.25$ & $2.1 \pm 0.14$ & $2.4 \pm 0.30$ \\
\hline $1.5+0.1$ & 70 & $4.2 \pm 0.15$ & $6.2 \pm 0.21$ & $3.4 \pm 0.15$ & $3.6 \pm 0.10$ & 70 & $4.2 \pm 0.14$ & $6.3 \pm 0.11$ & $2.6 \pm 0.24$ & $2.9 \pm 0.09$ & 75 & $4.4 \pm 1.02$ & $6.2 \pm 0.12$ & $3.3 \pm 0.30$ & $3.5 \pm 0.12$ \\
\hline $1.5+0.2$ & 55 & \begin{tabular}{|l|}
$3.1 \pm 0.48$ \\
\end{tabular} & $5.2 \pm 0.09$ & $2.9 \pm 0.32$ & $3.1 \pm 0.01$ & 60 & $3.2 \pm 0.22$ & $4.1 \pm 0.10$ & \begin{tabular}{|l|}
$2.4 \pm 0.31$ \\
\end{tabular} & $2.5 \pm 0.87$ & 45 & $3.1 \pm 0.61$ & $4.3 \pm 0.65$ & $2.9 \pm 0.54$ & $3.1 \pm 0.11$ \\
\hline $1.5+0.3$ & 40 & $2.4 \pm 0.17$ & $3.3 \pm 0.20$ & $2.7 \pm 0.74$ & $3.1 \pm 0.41$ & 50 & $3.1 \pm 0.15$ & $3.1 \pm 0.21$ & $2.2 \pm 0.35$ & $2.4 \pm 0.25$ & 30 & $2.2 \pm 0.11$ & $3.2 \pm 0.08$ & $2.7 \pm 0.54$ & $3.1 \pm 0.15$ \\
\hline $2+0.1$ & 60 & \begin{tabular}{|l|}
$5.3 \pm 0.21$ \\
\end{tabular} & $7.1 \pm 0.10$ & $3.4 \pm 0.08$ & $3.9 \pm 0.14$ & 65 & $5.2 \pm 0.41$ & $7.2 \pm 0.26$ & \begin{tabular}{|l|}
$3.1 \pm 0.05$ \\
\end{tabular} & $3.4 \pm 0.21$ & 60 & $3.3 \pm 0.95$ & $4.2 \pm 0.11$ & $3.4 \pm 0.18$ & $3.7 \pm 0.21$ \\
\hline $2+0.2$ & 45 & $4.2 \pm 0.31$ & $6.1 \pm 0.11$ & $3.1 \pm 0.70$ & $3.6 \pm 0.41$ & 45 & $4.3 \pm 0.01$ & $5.2 \pm 0.14$ & \begin{tabular}{|l|}
$2.8 \pm 0.07$ \\
\end{tabular} & $3.1 \pm 0.31$ & 40 & $3.2 \pm 0.01$ & $5.1 \pm 0.23$ & $3.1 \pm 0.72$ & $3.6 \pm 0.14$ \\
\hline $2+0.3$ & 40 & \begin{tabular}{|l|}
$4.1 \pm 0.45$ \\
\end{tabular} & $5.1 \pm 0.09$ & $3.3 \pm 0.10$ & $3.8 \pm 0.11$ & 30 & \begin{tabular}{|l|}
$3.1 \pm 0.21$ \\
\end{tabular} & $4.1 \pm 0.32$ & \begin{tabular}{|l|}
$2.7 \pm 0.16$ \\
\end{tabular} & $2.9 \pm 0.09$ & 40 & $2.2 \pm 1.12$ & $4.2 \pm .041$ & $3.3 \pm 0.57$ & $3.8 \pm 0.23$ \\
\hline \multicolumn{16}{|c|}{ BAP+NAA } \\
\hline $1+0.1$ & 40 & \begin{tabular}{|l|}
$3.2 \pm 0.11$ \\
\end{tabular} & $5.1 \pm 0.31$ & $2.3 \pm 2.20$ & $2.8 \pm 0.41$ & 20 & \begin{tabular}{|l|}
$2.1 \pm 0.05$ \\
\end{tabular} & $4.3 \pm 0.14$ & $2.2 \pm 0.21$ & $2.4 \pm 0.31$ & 20 & $2.1 \pm 0.58$ & $4.3 \pm 0.41$ & $2.2 \pm 0.54$ & $2.3 \pm 0.21$ \\
\hline $1+0.2$ & 45 & \begin{tabular}{|l|}
$2.1 \pm 0.08$ \\
\end{tabular} & $3.2 \pm 0.01$ & $2.2 \pm 0.26$ & $2.9 \pm 0.01$ & 25 & $2.3 \pm 0.00$ & $5.1 \pm 0.04$ & \begin{tabular}{|l|}
$2.2 \pm 0.04$ \\
\end{tabular} & $2.5 \pm 0.12$ & 25 & $2.2 \pm 0.31$ & $5.1 \pm 0.42$ & $2.2 \pm 0.46$ & $2.4 \pm 0.36$ \\
\hline $1+0.5$ & 50 & $2.3 \pm 0.00$ & $5.1 \pm 0.11$ & $2.1 \pm 0.42$ & $2.7 \pm 0.01$ & 30 & $3.3 \pm 0.45$ & $4.3 \pm 0.38$ & \begin{tabular}{|l|}
$2.3 \pm 1.20$ \\
\end{tabular} & $2.7 \pm 0.87$ & 30 & $3.3 \pm 0.37$ & $4.1 \pm 0.24$ & $2.3 \pm 0.38$ & $2.6 \pm 0.23$ \\
\hline $2+0.1$ & 60 & \begin{tabular}{|l|}
$4.3 \pm 1.02$ \\
\end{tabular} & $5.2 \pm .041$ & $3.4 \pm 0.95$ & $3.9 \pm 0.21$ & 30 & $2.2 \pm 0.04$ & $4.2 \pm 0.01$ & \begin{tabular}{|l|}
$2.4 \pm 0.33$ \\
\end{tabular} & $2.6 \pm 0.11$ & 40 & $2.3 \pm 1.00$ & $4.3 \pm 0.23$ & $2.4 \pm 0.56$ & $2.6 \pm 0.39$ \\
\hline $2+0.2$ & 70 & \begin{tabular}{|l|}
$4.3 \pm 0.43$ \\
\end{tabular} & $6.1 \pm 0.41$ & $3.1 \pm 0.29$ & $3.5 \pm 0.04$ & 45 & $3.5 \pm 0.00$ & $5.2 \pm 0.21$ & \begin{tabular}{|l|}
$2.5 \pm 0.39$ \\
\end{tabular} & $2.8 \pm 0.25$ & 40 & $3.1 \pm 0.03$ & $5.1 \pm 0.12$ & $2.5 \pm 0.12$ & $2.8 \pm 0.36$ \\
\hline $2+0.5$ & 60 & \begin{tabular}{|l|}
$3.1 \pm 0.31$ \\
\end{tabular} & $5.3 \pm 0.15$ & $3.2 \pm 0.45$ & $3.6 \pm 0.52$ & 50 & $3.1 \pm 0.61$ & $5.1 \pm 0.01$ & \begin{tabular}{|l|}
$2.5 \pm 0.04$ \\
\end{tabular} & $2.9 \pm 0.08$ & 60 & $3.2 \pm 0.30$ & $5.2 \pm 0.08$ & $2.5 \pm 0.09$ & $2.9 \pm 0.11$ \\
\hline $3+0.1$ & 80 & $5.4 \pm 0.14$ & $7.4 \pm 0.31$ & $3.6 \pm 0.01$ & $4.1 \pm 0.52$ & 70 & $4.4 \pm 0.01$ & $5.3 \pm 0.11$ & \begin{tabular}{|l|}
$2.6 \pm 0.09$ \\
\end{tabular} & $3.7 \pm 0.03$ & 70 & $4.4 \pm 0.36$ & $6.3 \pm 0.25$ & $3.2 \pm 0.07$ & $3.6 \pm 0.14$ \\
\hline $3+0.2$ & 60 & \begin{tabular}{|l|}
$4.1 \pm 0.05$ \\
\end{tabular} & $6.2 \pm 0.02$ & $3.1 \pm 0.54$ & $3.6 \pm 0.14$ & 55 & \begin{tabular}{|l|}
$3.1 \pm 0.00$ \\
\end{tabular} & $5.1 \pm 0.08$ & \begin{tabular}{|l|}
$2.5 \pm 0.62$ \\
\end{tabular} & $3.1 \pm 0.31$ & 60 & $3.2 \pm 0.11$ & $5.1 \pm 0.24$ & $3.1 \pm 0.54$ & $3.4 \pm 0.06$ \\
\hline $3+0.5$ & 50 & $5.1 \pm 0.84$ & $6.2 \pm 0.21$ & $3.3 \pm 0.39$ & $3.7 \pm 0.21$ & 50 & $2.2 \pm 1.00$ & $4.2 \pm 0.47$ & \begin{tabular}{|l|}
$3.3 \pm 0.32$ \\
\end{tabular} & $3.7 \pm 0.01$ & 50 & $2.3 \pm 0.01$ & $4.2 \pm 0.15$ & $3.3 \pm 0.35$ & $3.5 \pm 0.18$ \\
\hline
\end{tabular}

